\title{
AIAA 96-1806
}

The Study of Flow Pattern And Phase-Change Problem in Die Casting Process

H. Wei, Y. S. Chen and H. M. Shang

Engineering Sciences, Inc.

1900 Golf Road, Suite D

Huntsville, AL 35802

T. S. Wang

NASA-Marshall Space Flight Center

Huntsville, AL 35812

\section{1st AIAA Thermophysics Conference June 17-20, 1996 / New Orleans, LA}




\title{
THE STUDY OF FLOW PATTERN AND PHASE-CHANGE PROBLEM IN DIE CASTING PROCESS
}

\author{
H. Wei*, Y. S. Chen ${ }^{\dagger}$, H. M. Shang ${ }^{*}$ \\ Engineering Sciences, Inc, Huntsville, Alabama \\ and \\ T. S. Wang ${ }^{\S}$ \\ NASA-Marshall Space Flight Center, Huntsville, Alabama
}

\begin{abstract}
The flow pattern and solidification phenomena in die casting process have been investigated in the first phase study. The flow pattern in filling process is predicted by using a VOF method. A good agreement with experimental observation is obtained for filling the water into a die cavity with different gate geometry and with an obstacle in the cavity. An enthalpy method has been applied to solve the solidification problem. By treating the latent heat implicitly into the enthalpy instead of explicitly into the source term, the CPU time can be reduced at least 20 times. The effect of material properties on solidification fronts is tested. It concludes that the dependence of properties on temperature is significant. The influence of the natural convection over the diffusion has also been studied. The result shows that the liquid metal solidification phenomena is diffusion dominant, and the natural convection can affect the shape of the interface. In the second phase study, the filling and solidification processes will be considered simultaneously.
\end{abstract}

\section{INTRODUCTION}

Due to its importance in manufacturing, analysis of casting process for metals has received considerable attention in recent years. For improving the quality of manufactural parts with minimal experimentation, it is important to understand how the transport phenomena could affect the microstructure. Numerical analysis and

\footnotetext{
-Research Scientist, Engineering Sciences, Inc, Member AIAA ${ }^{+}$Presendent, Engineering Sciences, Inc, Senior Member AlAA

"Senior Research Scientist, Engineering Science, Inc, Member AlAA

Researcher, CFD Branch, Member AlAA

Copyrighı $C 1996$ by the American Institute of Aeronautics and Astronautics, Inc All rights resened
}

modeling efforts provide a powerful means to achieve the goal.

For simplifying the problem, die casting process can be basically divided into the filling stage and solidification stage. It can be assumed that no phase change in the filling stage and no forced flow when phase change started, because the filling is much faster than solidification. The study of both of these two phenomena is presented in this paper, and the combined process will be investigated in the next step of the study.

The fluid flow in filling stage involves a free surface between two different fluids (gas and liquid). The volume of fluid (VOF) method developed in SOLA-VOF ${ }^{1}$ is used here and the numerical implementation details can be found in Ref. [2]. The surface tension force is calculated by Continuum Surface Force (CSF) model ${ }^{3}$, in which the surface tension is treated as a body force.

The solidification is handled by an enthalpy model, which is initially developed by Shamsunder and Sparrow ${ }^{4}$. In this model, the enthalpy is used as a dependent variable. The liquid phase, solid phase and the interface all satisfy a unique governing equation. This can be solved like a single phase problem, and no interface tracking necessary. After the enthalpy field is solved at every time step, the temperature and the interface position can be obtained from the enthalpytemperature diagram. Voller and coworkers ${ }^{5.7}$ improved this model by applying a porosity concept to identify the phase change region, which forms a so-called enthalpy-porosity model. In this model, the latent heat content, $\Delta \mathrm{H}$, varies among 0 (cell all solid) and $\mathrm{L}$ (cell all liquid), where $\mathrm{L}$ is the latent heat of the phase change. The porosity $\lambda$ is defined as the ratio of $\Delta H$ over $L$, then $\lambda$ decrease from 1 to 0 when phase changes from liquid to solid. So, by prescribing a "Darcy" source term in the momentum equation, which vanishes when $\lambda$ is 0 and increases rapidly when $\lambda$ approaches to 1 , the velocity will be forced to 0 when total 
solidification is achieved. All the previous calculations in Ref. [5-7] put the latent heat term explicitly into the source. In turn, the iteration is necessary in every time step for updating the latent heat content, so that the temperature in the cell that is undertaking phase change will keep its melting temperature. As the experience of the authors and as pointed in Ref. [6], the average iteration times is about $30-40$ per time step. This is very time consuming and expensive, which will make the 3D simulation unpracticable. In this paper, the latent heat will be included in the enthalpy and no iteration is needed, while the CPU time will decrease at least 20 times with the only extra effort of getting the temperature from enthalpy.

\section{GOVERNING EQUATIONS}

The die casting process can be assumed as a Newtonian fluid laminar flow. No turbulence is considered because the duration time of this transient process is very short. The governing equations in 2-D Cartesian coordinates are as follows.

Continuity equation:

$$
\frac{\partial \rho}{\partial}+\frac{\partial \rho u_{j}}{\partial x_{j}}=0
$$

Momentum equation in $\mathrm{x}$-direction:

$$
\frac{\partial \rho u}{\partial}+\frac{\partial \rho u_{j} u}{\partial x_{j}}=-\frac{\partial P}{\partial x}+\frac{\partial}{\partial x_{j}}\left(\mu \frac{\partial u}{\partial x_{j}}\right)+S_{x}+F_{s x}
$$

Momentum equation in y-direction:

$$
\begin{aligned}
& \frac{\partial \rho v}{\partial t}+\frac{\partial \rho u_{j} v}{\partial x_{j}}=-\frac{\partial P}{\partial y}+\frac{\partial}{\partial x_{j}}\left(\mu \frac{\partial v}{\partial x_{j}}\right) \\
& +S_{y}+S_{f}+F_{s y}
\end{aligned}
$$

Energy equation:

$$
\frac{\partial \rho H}{\partial}+\frac{\partial \rho u_{j} H}{\partial x_{j}}=\frac{\partial}{\partial x_{j}}\left(k \frac{\partial T}{\partial x_{j}}\right)-S_{h}
$$

In filling stage, the free surface exists and the surface tension force terms $F_{s x}$ and $F_{s y}$ should be include in the momentum equations. It will be discussed in the CSF model. The $S_{f}$ term is gravity force $-\rho g$ and the "Darcy" source terms $S_{x}$ and $S_{y}$ are zero. The energy equation does not need to be solved for this isothermal process.

In the solidification stage, all the properties, including density, viscosity and thermal conductivity, are constants when using the incompressible flow approach. The natural convection is generated by the buoyancy force using Boussinesq approximation.

$$
S_{f}=\rho g \beta\left(T-T_{\text {ref }}\right)
$$

Where, $\beta$ is thermal expansion coefficient and $T_{r e f}$ is the reference temperature. Because the temperature range is very large in solid phase (from room temperature to melting temperature), the dependence of properties on temperature can not be ignored. In this case, the density, viscosity, thermal conductivity and heat capacity will be updated on every time step according to the temperature. The $S_{f}$ term will just be a gravity force $-\rho g$. The "Darcy" source terms $S_{x}, S_{y}$ and the energy source term $S_{h}$ will be given later in the enthalpy-porosity model.

\section{VOF METHOD and CSF MODEL}

In the filling stage of casting process, the position and shape of the free surface between the liquid and gas phase can be represented by the VOF values. The fractional volume in a typical control volume cell is defined as:

$$
F=\frac{V_{l}}{V_{g}+V_{l}}
$$

Where, $\mathrm{V}$ represents volume occupied by gas $\left(\mathrm{V}_{\mathrm{g}}\right)$ and liquid $\left(V_{D}\right)$ within the control volume considered. The function $F$ obeys the volume flux conservation equation:

$$
\frac{\partial F}{\partial t}+\frac{\partial u_{j} F}{\partial x_{j}}=0
$$

The average density is then defined as

$$
\rho=\rho_{l} F+\rho_{g}(1-F)
$$


The surface tension is an inherent characteristic of material interface, because fluid molecules at or near the surface experience uneven molecular forces of attraction. CSF model interprets the surface tension force as a continuous, three-dimensional effect across an interface, and the interface reconstruction is no longer needed.

$$
F_{s}=\sigma \kappa \nabla F
$$

Where $\sigma$ is the fluid surface tension coefficient (in units of force per unit length), $F$ is the VOF value and $\kappa$ is the local surface curvature.

$$
\kappa=-\nabla \cdot\left(\frac{\nabla F}{|\nabla F|}\right)
$$

\section{ENTHALPY-POROSITY MODEL}

Voller ${ }^{5.7}$ improved the enthalpy model by introducing the porosity concept, which forms the enthalpy-porosity model. In this model, an unique energy equation is suitable for the whole calculation domain and no liquid-solid interface need to be accounted for. The zero velocity condition in the vicinity of the interface is modeled by a "Darcy" source term in momentum equation.

Voller substitutes the $\mathrm{H}=\mathrm{cT}+\Delta \mathrm{H}$ into the energy equation and moves the time variation and convection term of $\Delta \mathrm{H}$ to the right hand side, which forms (relate to Equation (4))

$$
\begin{gathered}
H=c T \\
S_{h}=\frac{\partial \rho \Delta H}{\partial}+\frac{\partial}{\partial x_{j}}\left(\rho u_{j} \Delta H\right)
\end{gathered}
$$

In the approach of this paper, however, $\Delta \mathrm{H}$ is implicitly included in the enthalpy so that

$$
\begin{gathered}
H=c T+\Delta H \\
S_{h}=0
\end{gathered}
$$

After the enthalpy field is obtained, the temperature and the porosity can be solved from enthalpy-temperature diagram:

$$
\begin{aligned}
& \text { If } T<T_{m}-\varepsilon, \\
& \text { then } H=c_{s} T, \quad \Delta H=0 \\
& \text { If } T_{m}-\varepsilon<T<T_{m}+\varepsilon, \\
& \text { then } H=c_{s} T+\Delta H, \quad 0<\Delta H<L \\
& \text { If } T>T_{m}+\varepsilon, \\
& \text { then } H=c_{s} T+L+c_{l}\left(T-\left(T_{m}+\varepsilon\right)\right) \text {, } \\
& \Delta H=L
\end{aligned}
$$

The subscripts s, I denote solid phase and liquid phase respectively. If the material is not pure (e.g. alloy), the phase change takes place over a temperature range, call mushy region $\left(T_{m}-\varepsilon\right.$ to $T_{m}+\varepsilon$ ), where $T_{m}$ refers to the center of the mushy region. For isothermal phase change problem (pure material), $\mathrm{T}_{\mathrm{m}}$ is the melting temperature and $\varepsilon$ equal to zero. The problem considered in this study is isothermal phase change.

The "Darcy" source terms in momentum equations are constructed as:

$$
\begin{aligned}
& S_{x}=A u \\
& S_{y}=A v
\end{aligned}
$$

Where

$$
A=-\frac{D(1-\lambda)^{2}}{\left(\lambda^{3}+d\right)}
$$

The porosity $\lambda$ is:

$$
\lambda=\frac{\Delta H}{L}
$$

The constant $D$ is very large that can force the velocity effectively to zero when the cell is total solidified. Constant $d$ is a small number for avoiding divided by zero. In this study, $\mathrm{D}$ and $\mathrm{d}$ are chosen as $D=1.6 \times 10^{5}$ and $d=0.1$ according to Ref. [5]. The parameter A is formulated in such a way that the "Darcy" source term has no any influence in the fully liquid cells. While it gradually increase, dominate and finally override all other terms in the momentum equation when solid portion increases in that cell. This smooth transition is superior than the step changes that may cause divergence.

\section{NUMERICAL METHOD}


The flow solver used in this study is the FDNS code (Finite Difference Navier Stokes Solver). FDNS code can be used to solve 2-D planner, 2-D axisymmetric or 3-D forms of NavierStokes equations and other scale transport equations using primitive variables and curvilinear coordinate with non-staggered mesh systems. A pressure based predictor/multi-corrector solution procedure is employed in the FDNS code to enhance velocity-pressure coupling and massconserved solution at the end of each time step. This pressure based method is suitable for all speed flow computations including supersonic and subsonic flow, compressible and incompressible flow, laminar and turbulent flow. The detailed description of FDNS code can be found in Ref. [8, 9].

\section{RESULTS and DISCUSSIONS}

The water experiments ${ }^{10,11}$ during the filling stage are calculated here for flow pattern comparison. The dimension of the die is $152 \times 102 \times 3 \mathrm{~mm}$ with the gates and obstacle having the same thickness as the die $(3 \mathrm{~mm})$. A uniform $61 \times 61$ grid mesh is used in all the calculations. The gravity force is considered downwards. As pointed in Ref. [7] that due to gate velocity in the experiment may vary with time and the calculation assume constant inlet velocity, the comparison of the prediction and the experiment is made at the same percentage filling of cavity.

Figure 1 is for one gate case, where the gate is located on $3 \mathrm{~mm}$ from the bottom of the right wall. The gate width is $6 \mathrm{~mm}$, and the gate velocity is $10.7 \mathrm{~m} / \mathrm{s}$ which corresponding to the Reynolds number of $6.4 \times 10^{4}$. The void in the left bottom corner is caused by the recirculation and reattachment of the fluid on the bottom wall. A very fine grid mesh is needed to capture this void during the whole process.

Figure 2 is for the case of two inflow gates, where one gate is at the bottom right wall and another is at the bottom left wall. The gate width is $12 \mathrm{~mm}$, and the gate velocity is $15.8 \mathrm{~m} / \mathrm{s}$ which corresponding to the Reynolds number of $1.9 \times 10^{5}$. The two incoming flows impinge on and go upward, and then travel along the walls. The numerical predictions are verified by the experiments.

Figure 3 is the case with an obstacle inside the cavity, where the gate is at center of the bottom wall. The gate width is $12 \mathrm{~mm}$, and the gate velocity is $18.3 \mathrm{~m} / \mathrm{s}$ which corresponding to the Reynolds number of $2.2 \times 10^{5}$. The obstacle is $50 \times 25 \mathrm{~mm}$ located $25 \mathrm{~mm}$ above the gate. The figure illustrates that two recirculation zones are generated and preserved near the bottom left and right comers. Other two separation zones are present at the left and right walls of obstacle near the end of filling. The calculated flow patterns agree with the experimental observations very well.

Table 1. Thermophysical properties of aluminum

\begin{tabular}{|c|c|}
\hline$\rho\left(\mathrm{kg} / \mathrm{m}^{3}\right)$ & $2.38 \times 10^{3}$ \\
\hline$\mu(\mathrm{kg} / \mathrm{m} \mathrm{s})$ & $2.9 \times 10^{-3}$ \\
\hline$\beta(1 / \mathrm{K})$ & $1.16 \times 10^{-4}$ \\
\hline $\mathrm{c}(\mathrm{J} / \mathrm{kg} \mathrm{K})$ & $1.08 \times 10^{3}$ \\
\hline $\mathrm{k}(\mathrm{W} / \mathrm{m} \mathrm{K})$ & $1.03 \times 10^{2}$ \\
\hline $\mathrm{L}(\mathrm{J} / \mathrm{kg})$ & $4 \times 10^{3}$ \\
\hline
\end{tabular}

Figures 4-7 represent the results of solidification of liquid aluminum. The liquid aluminum is initially $981.7 \mathrm{~K}(50 \mathrm{~K}$ superheat) inside a $150 \times 150 \mathrm{~mm}$ cavity. At the beginning of the solidification, the left wall is lowered to $291.7 \mathrm{~K}$ (640 K below melting point) and other three walls are adiabatic. Figure 4 shows the moving interfaces and liquid flow patterns for the constant properties approach, where the properties are given in Table 1. Case (a) considered the diffusion only and case (b) involved the natural convection also. Figure 5 is for the variable properties approach, where the density, viscosity, thermal conductivity and heat capacity are updated according to the temperature at every time step. Same as in Figure 4, case (a) considered the diffusion only and case (b) involved the natural convection also. Comparing case (a) with case (b) in Figures 4 and 5, one can see that the conduction dominates the solidification process, and the natural convection acts on it by effecting the shape of the interface. Because the hot fluid goes upward and cold fluid goes downward, the solid front goes faster at the bottom than it does at the top. The comparison of Figure 4 and Figure 5 shows that the dependence of the material properties on temperature cannot be ignored. For more clear demonstrating this influence, the positions of solid front at the bottom wall for case (b) are drawn in Figure 6. The difference can reach $50 \%$ for this particular case. The main reason for such a large effect is that the temperature changes $640 \mathrm{~K}$ inside the solid phase, so that the consideration of the 
thermal conductivity and heat capacity as functions of temperature will largely enhance the heat conduction.

Except accounting for the property effects, the main contribution of this paper is that one algorithm is developed to solve the energy equation by including the latent heat inside the enthalpy instead of in source as did in Ref. [5-7]. Because the iteration is not needed in each time step, this method saves CPU time by more than 20 times. As shown in Figure 7, the correctness of this method has been justified by comparing the interface positions carried out by this method with the theoritical predictions ${ }^{12}$ for the case of purely conduction with constant properties. When considering the convection, the phase front has been compared with the calculation in Ref. [7], as shown in Figure 6. These two methods got same solid front position at 28 second after the beginning of solidification.

\section{CONCLUSIONS}

In the liquid filling analysis, flow patterns of different gate position and with or without inside obstacle have been compared to the experimental observations. The good agreement shows that the numerical predictions can provide useful information of the filling patterns and the order of different areas in cavity to be filled. The accurate predictions of the filling stage establish a basic foundation for further resolving the solidification and finally the die casting process.

In the investigation of the solidification, the effect of the variation of thermophysical properties as functions of temperature has been tested. This effect is very large due to the temperature range is very big for liquid metal solidification applications. It greatly reduces CPU time if solving energy equation by including the latent heat into the enthalpy instead of put it in the source term. This makes the simulation of 3-D real applications possible.

\section{REFERENCE}

1. Nicholes,B. D., Hirt, C. W., and Hotchkiss, R. S.: "SOLA-VOF: A Solution Algorithm for Transient Fluid Flow with Incompressible Flows with Free Surface," Los Alamos National Lab., LA-8355, Aug., 1980.
2. Chen, Y. S., Shang, H. M., Liaw, P., Chen, C. P., and Wang, T. S.: " A Unified Two-Phase Numerical Method for General Gas-Liquid Flow Applications," Gas-Liquid Flows, FEDVol. 25, pp. 99-106, Symposium of the Joint ASME/JSME Fluids Engineering Conference, August 13-18, 1995, Hilton Heat Island, SC.

3. Backbill, J. U., Kothe, D. B., and Zemach, C.: "A Continuum Method for Modeling Surface Tension," Journal of Computational Physics, Vol. 100, pp. 335-354, 1992.

4. Shamsundar, N., and Sparrow, E. M.: "Analysis of Multidimensional Conduction Phase Change Via the Enthalpy Model," Trans. of the ASME, Journal of Heat Transfer, pp. 333-340, Aug. 1975.

5. Voller, V. R., and Prakash: "A fixed grid numerical modeling methodology for convection-diffusion mushy region phasechange problems," International Journal of Heat and Mass Transfer, Vol. 30, No. 8, pp. 17091719, 1987.

6. Brent, A. D., Voller, V. R., and Reid, K. J.: "Enthalpy-Porosity Technique for Modeling Convection-Diffusion Phase Change: Application to the Melting of A Pure Metal," Numerical Heat Transfer, Vol. 13, pp. 297-318, 1988.

7. Minaie, B., Stelson, K. A., and Voller, V. R.: "Analysis of Flow Patterns and Solidification Phenomena in the Die Casting Process," Trans. of the ASME, Journal of Heat Transfer, Vol. 113, pp. 296-302, July, 1991.

8. Chen, Y. S.: "FDNS: A General Purpose CFD Code, User's Guide," Engineering Sciences, Inc., Huntsville, Alabama.

9. Chen, Y. S., Liaw, P., and Shang, H. M.: "Numerical Analysis of Complex Internal and External Viscous Flows with A Second-Order Pressure-Based Method," AIAA Paper 932966, 1993.

10. Smith, W. E., and Wallace, J. F.: "Gating of Die Castings," Trans. of AFS, Vol. 71, pp. 325$348,1963$.

11. Stuhrke, W. F., and Wallace, J. F.: "Gating of Die Castings," Trans. of AFS, Vol. 72, pp.374407, 1964.

12. Gebhart, Benjamin: Heat Conduction and Mass Diffusion, McGraw-Hill Series in Mechanical Engineering, McGraw-Hill, Inc., 1993. 

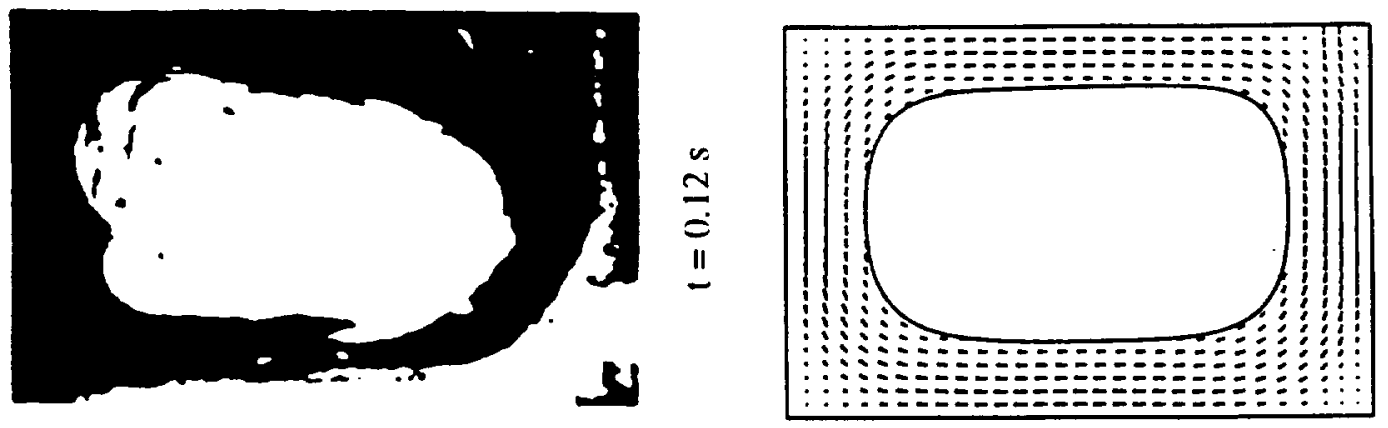

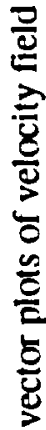
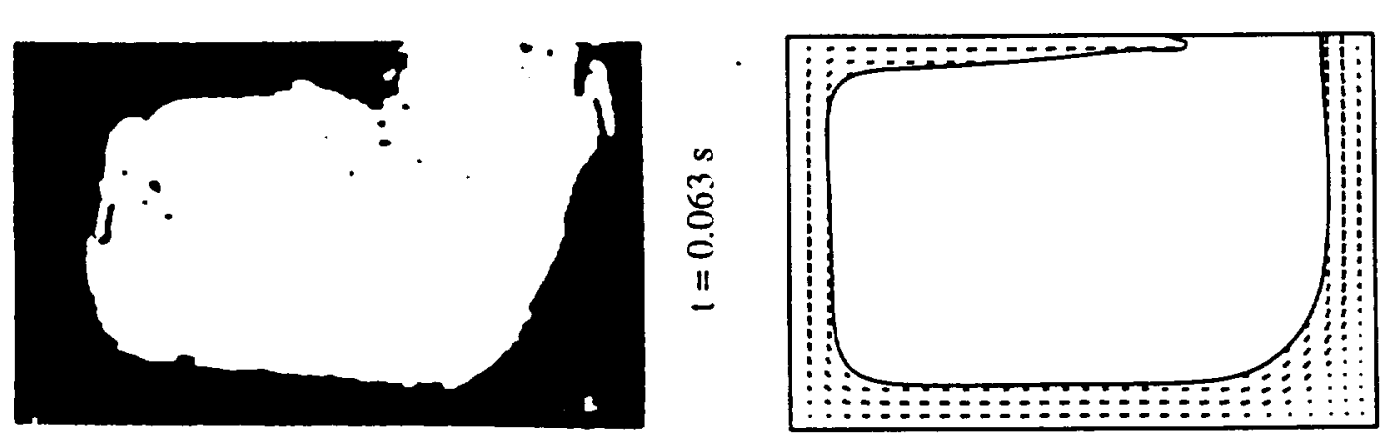

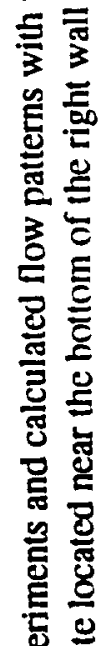
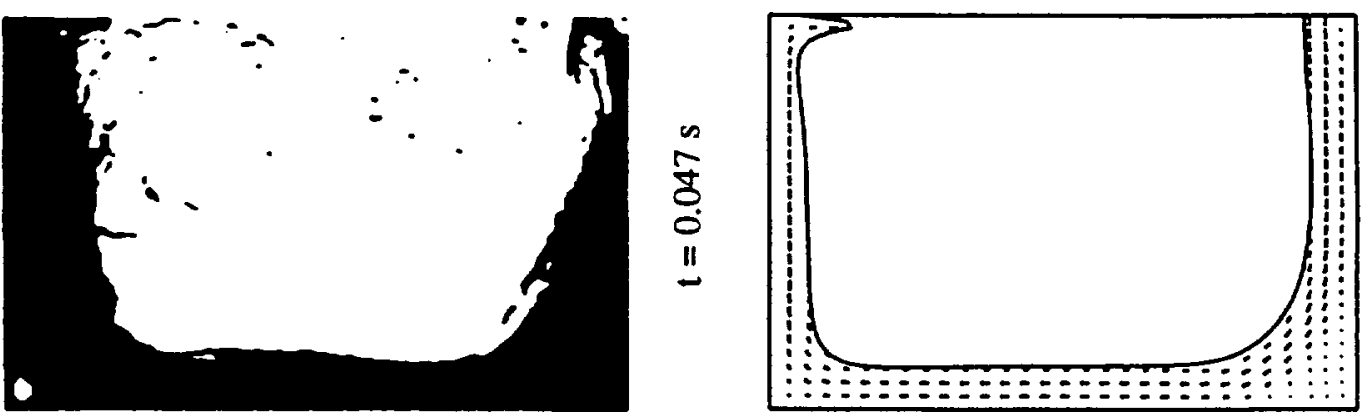

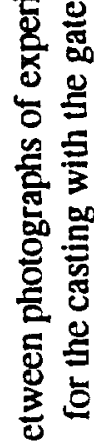
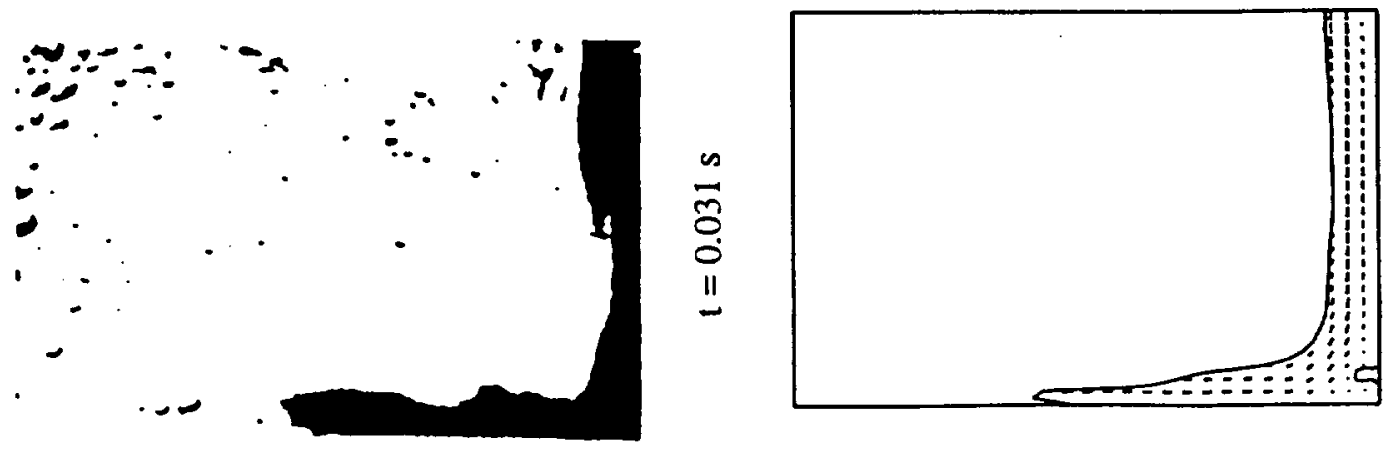

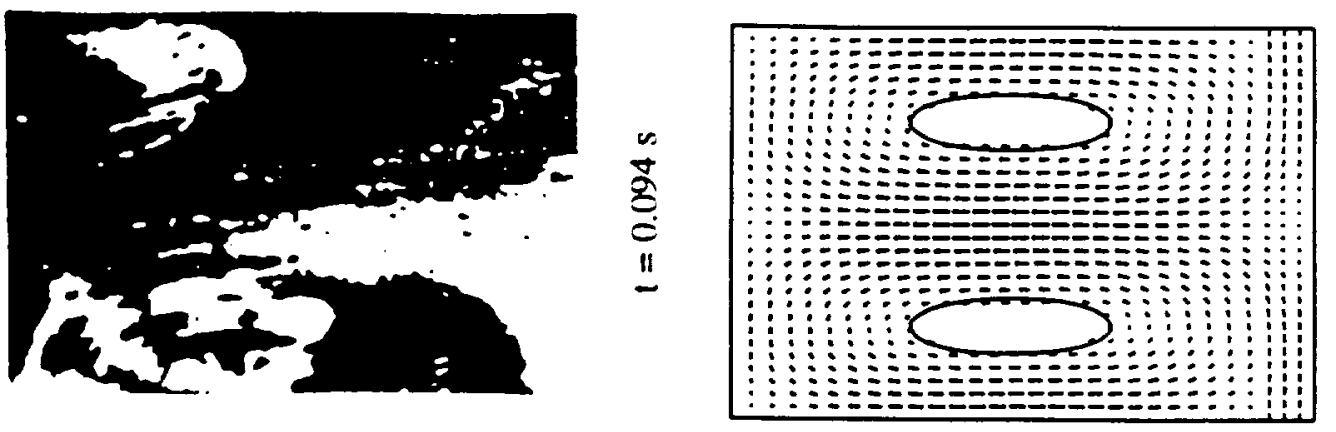

$\frac{2}{\frac{0}{0}}$
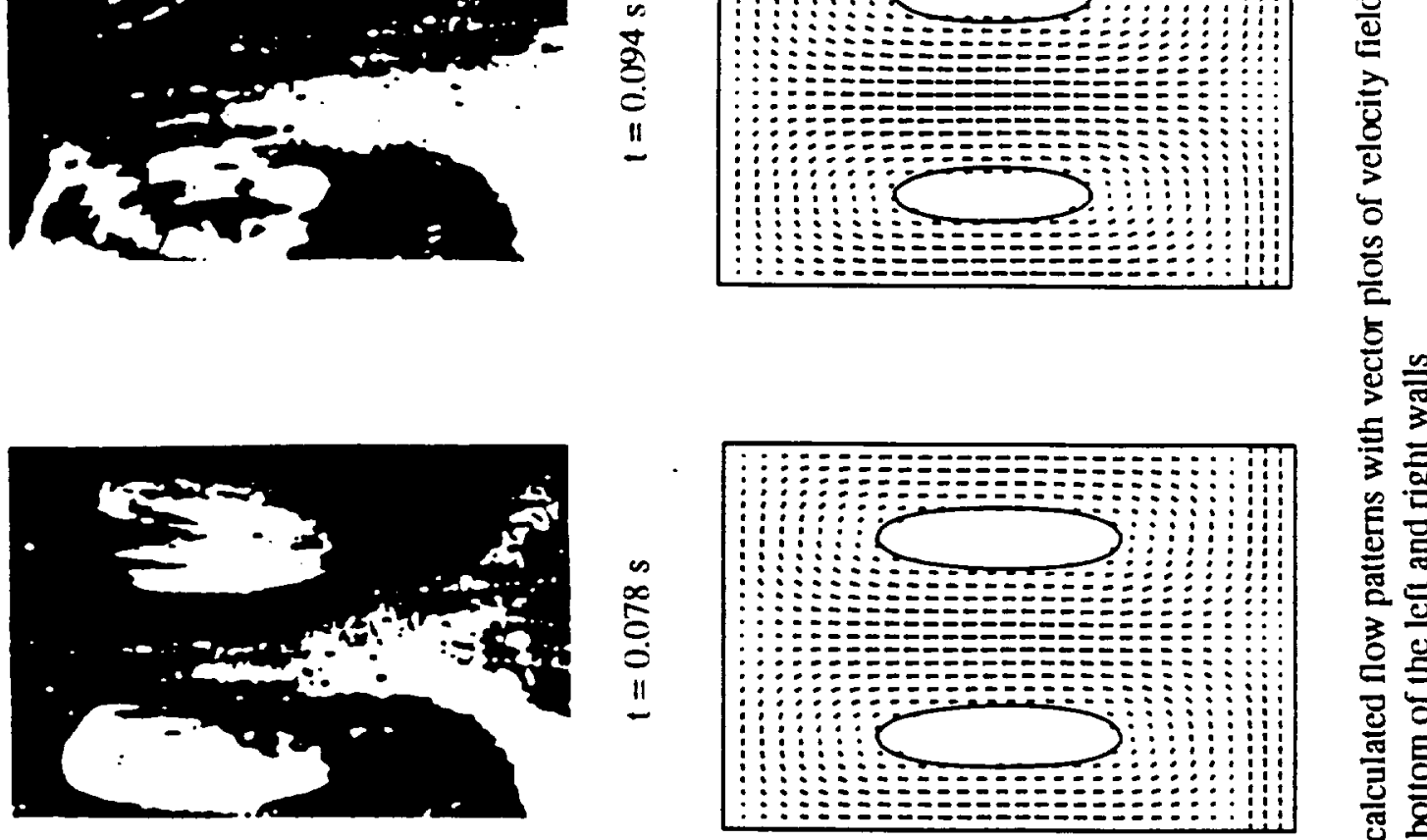

을

क त

를
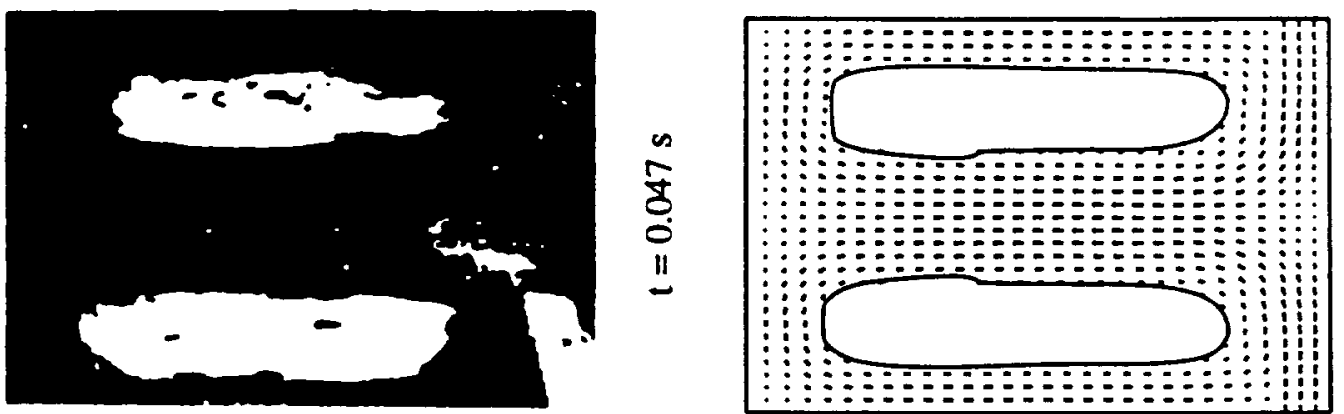

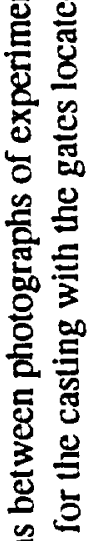

官
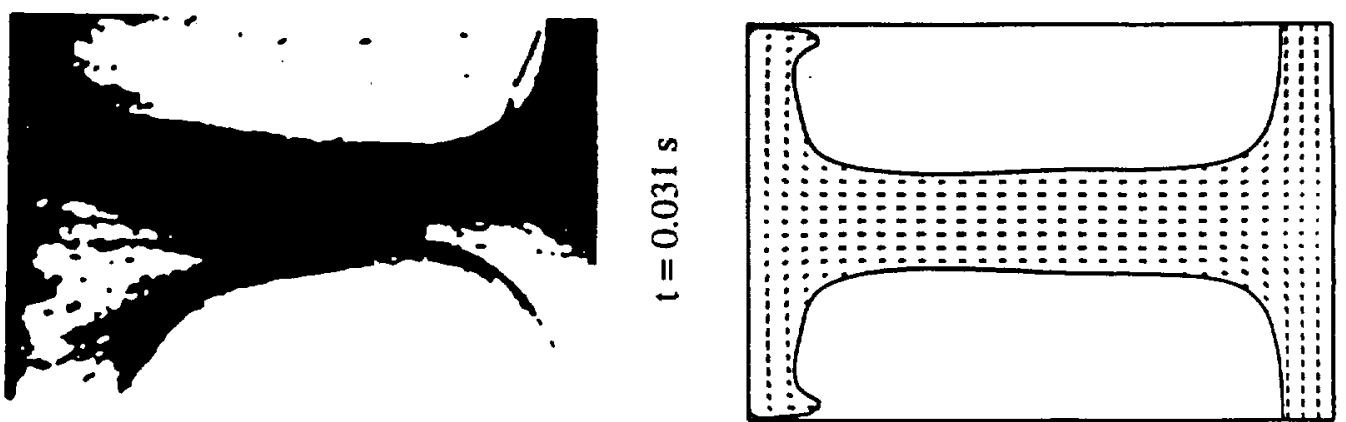

$\ddot{i}$

氜 

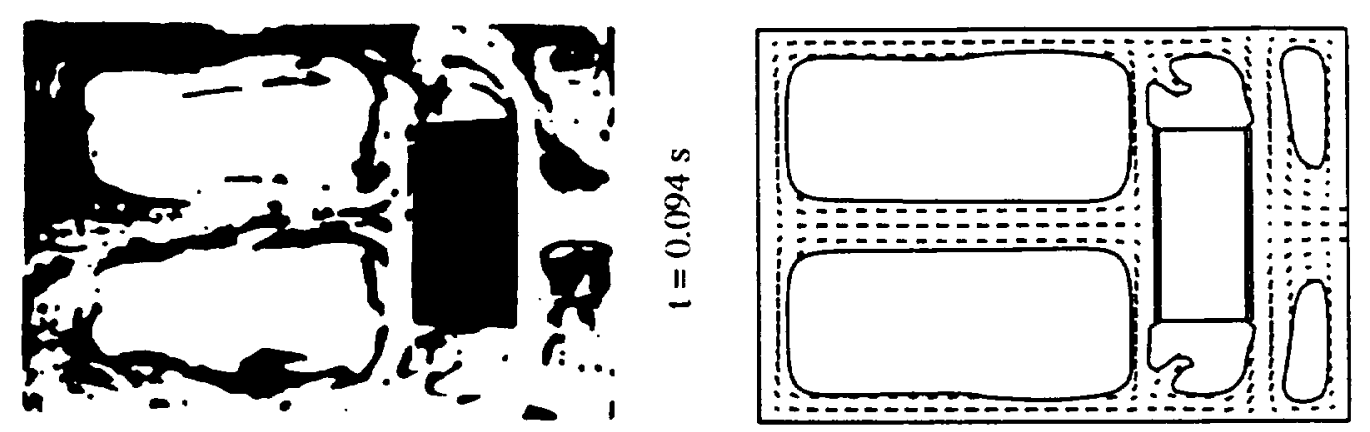

를
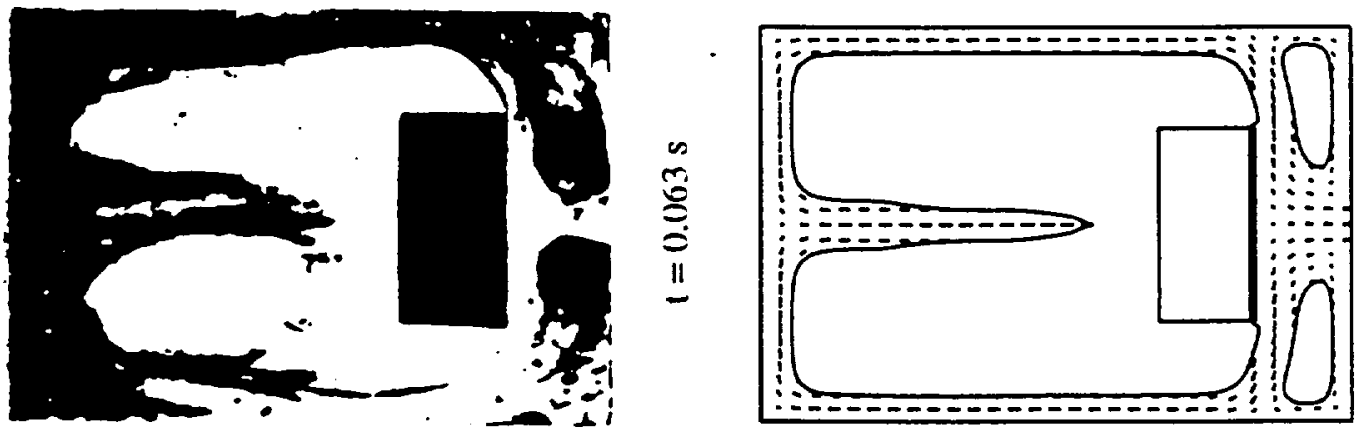

冚

它

$\equiv$

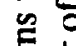

冚

3 U

产

异

电
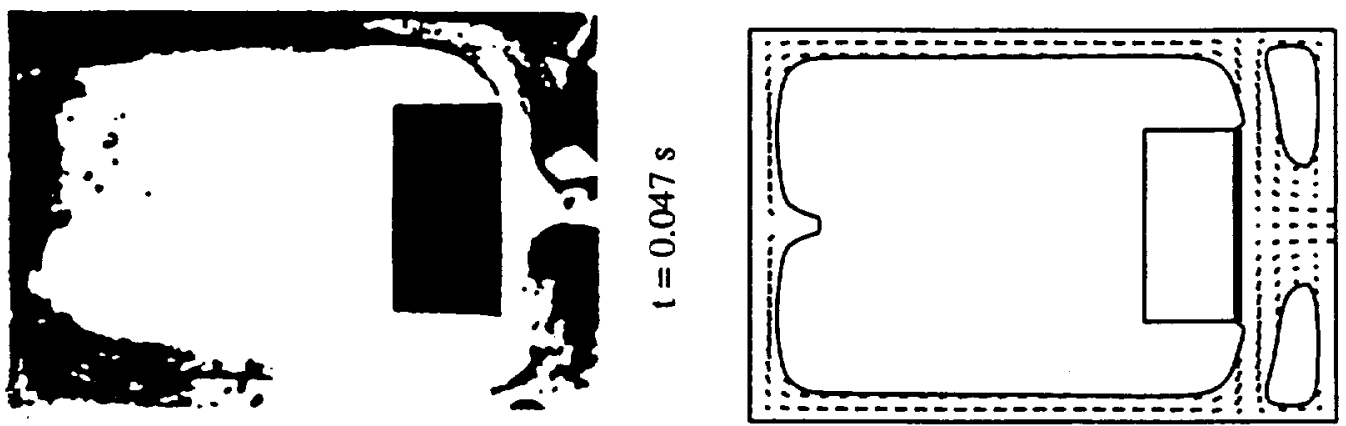

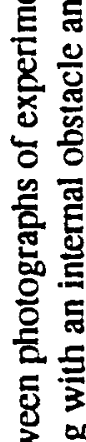

ฮั

号
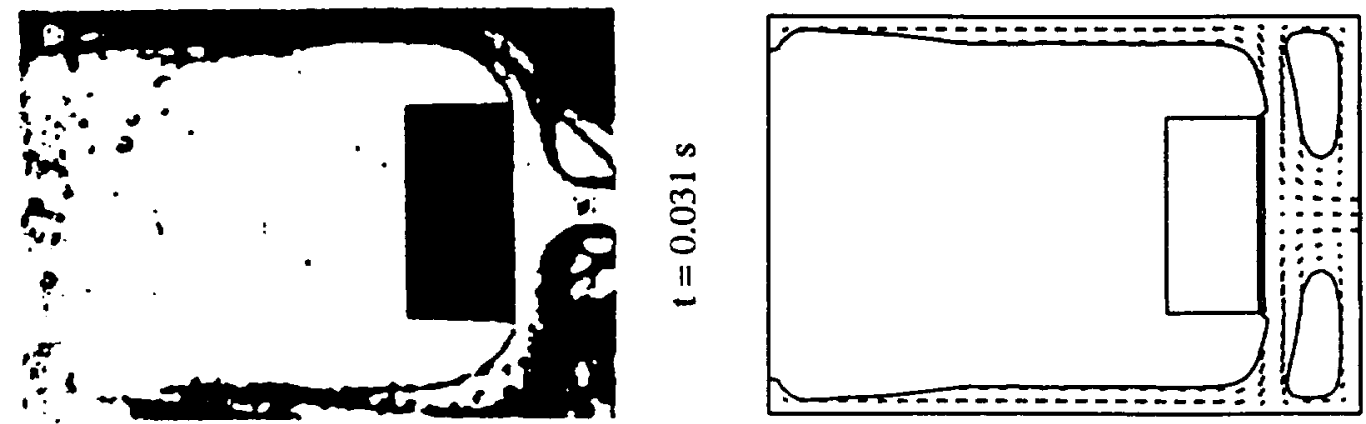

。

홍

豆

$\dot{m}$

氞 

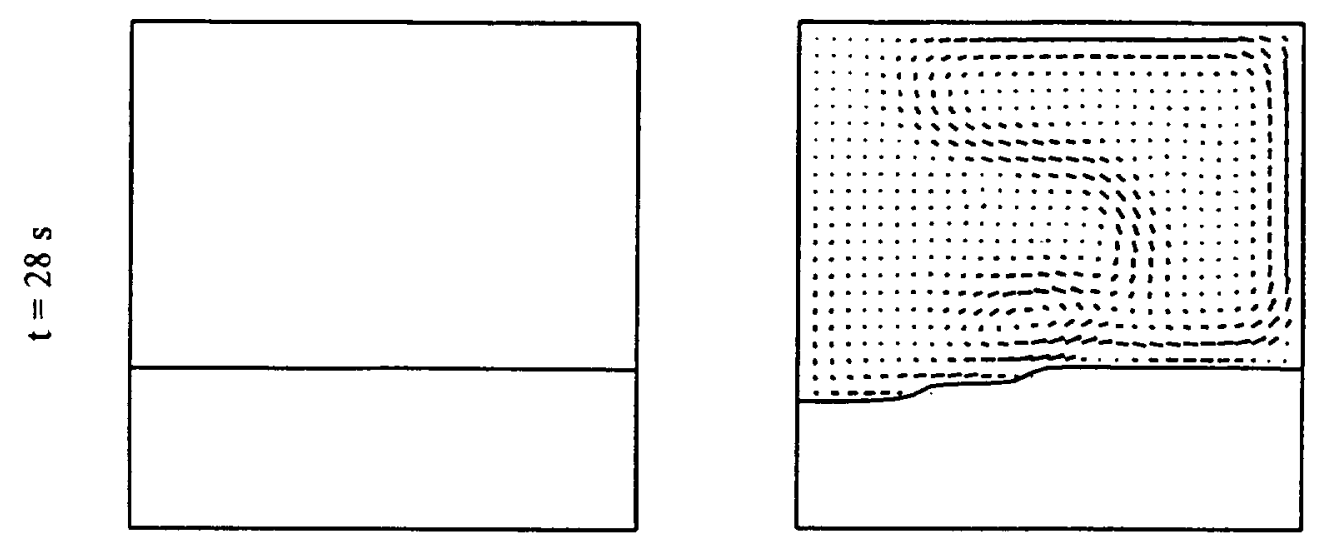

过
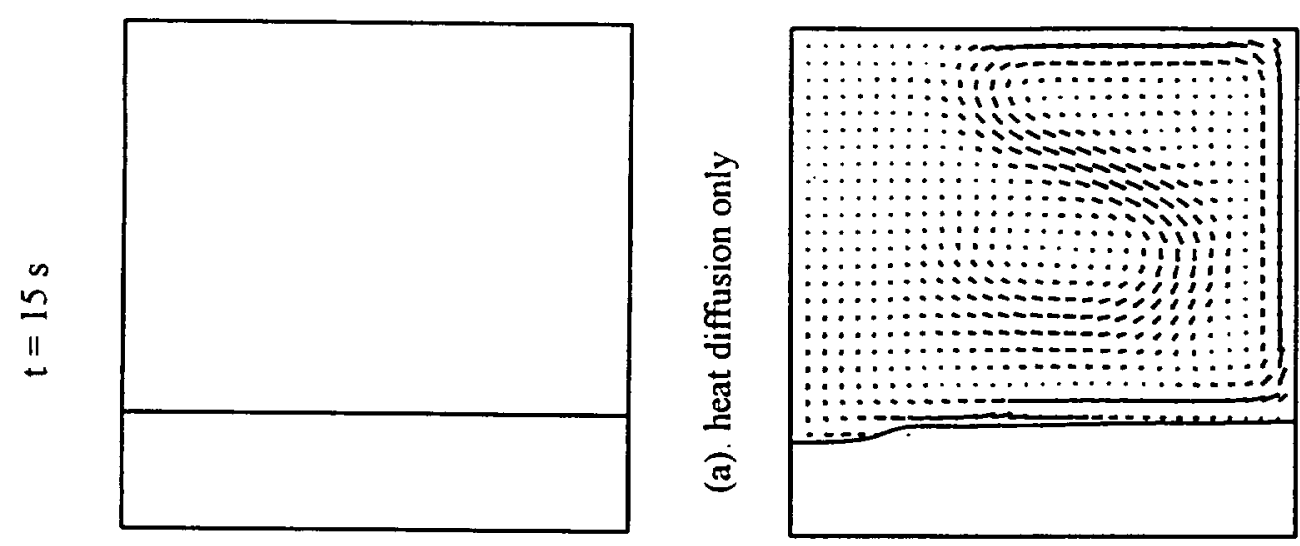

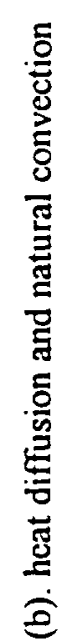

을
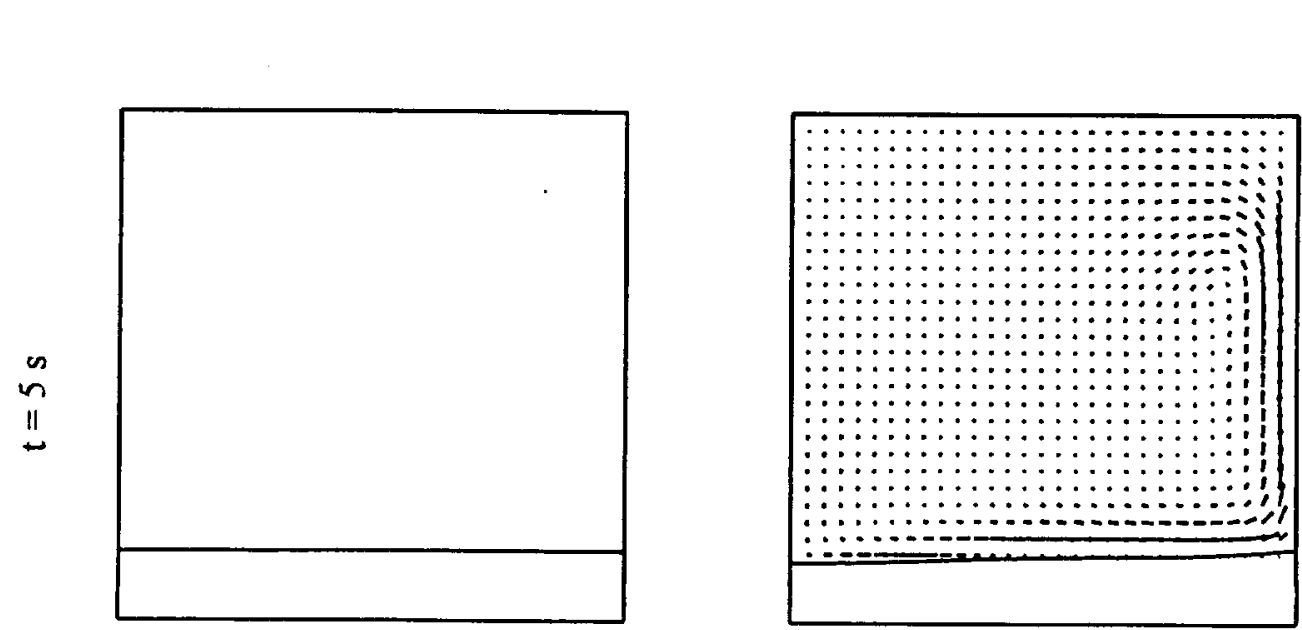

葛 

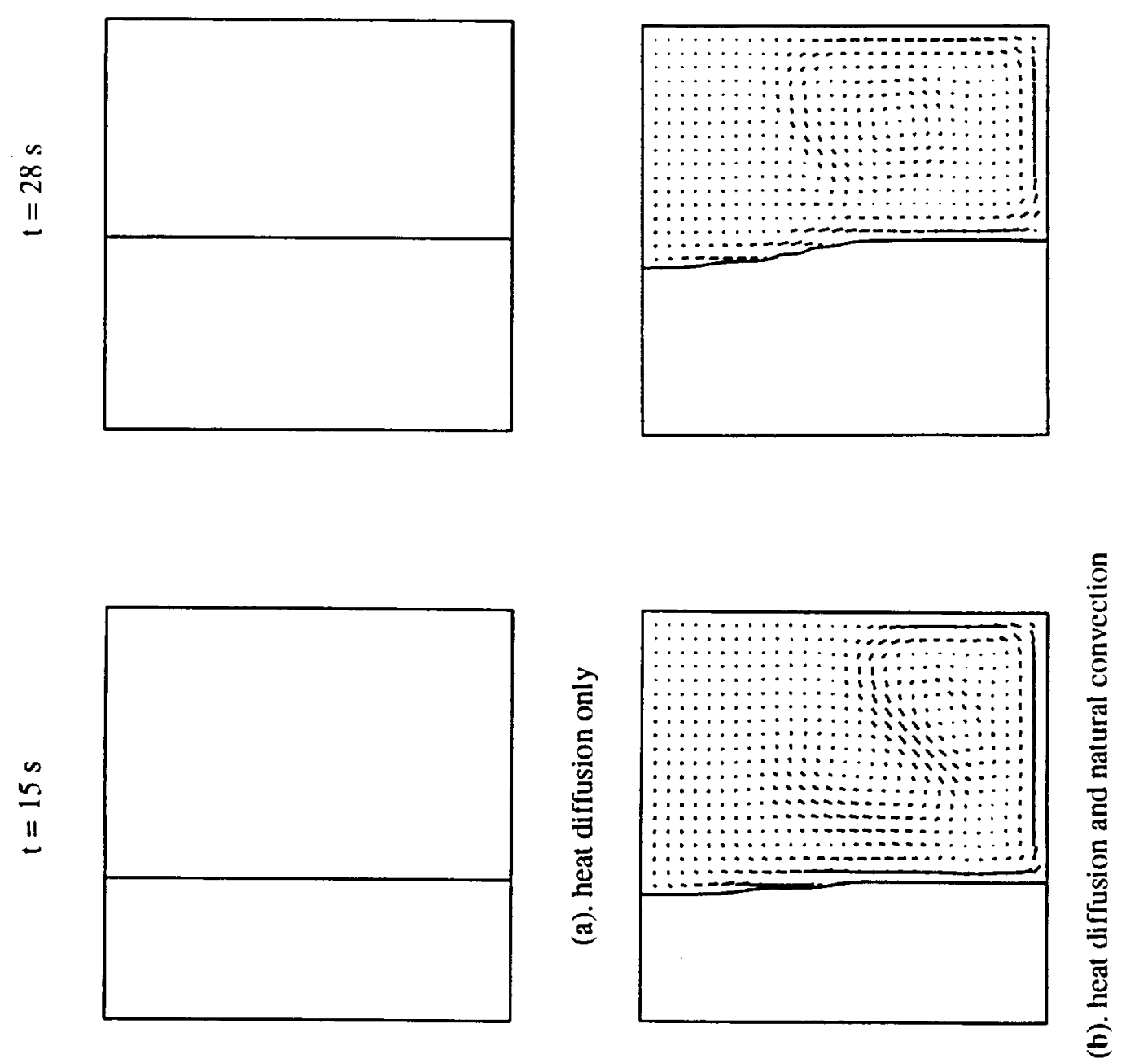

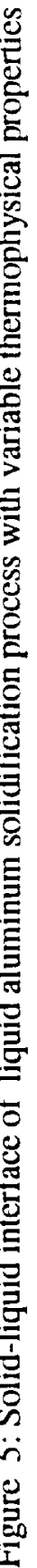
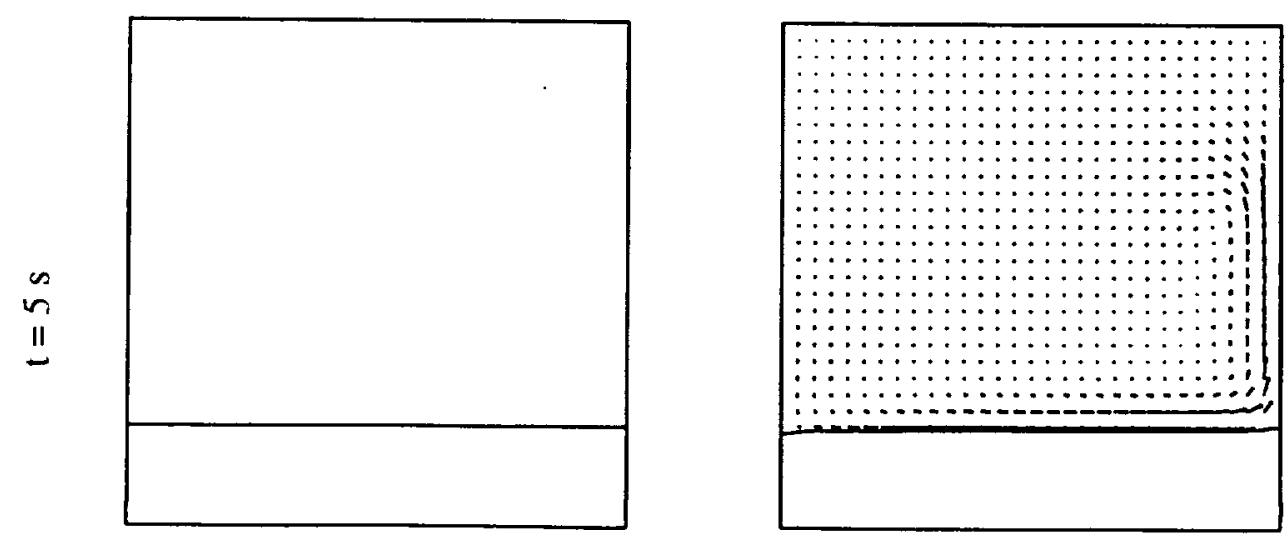


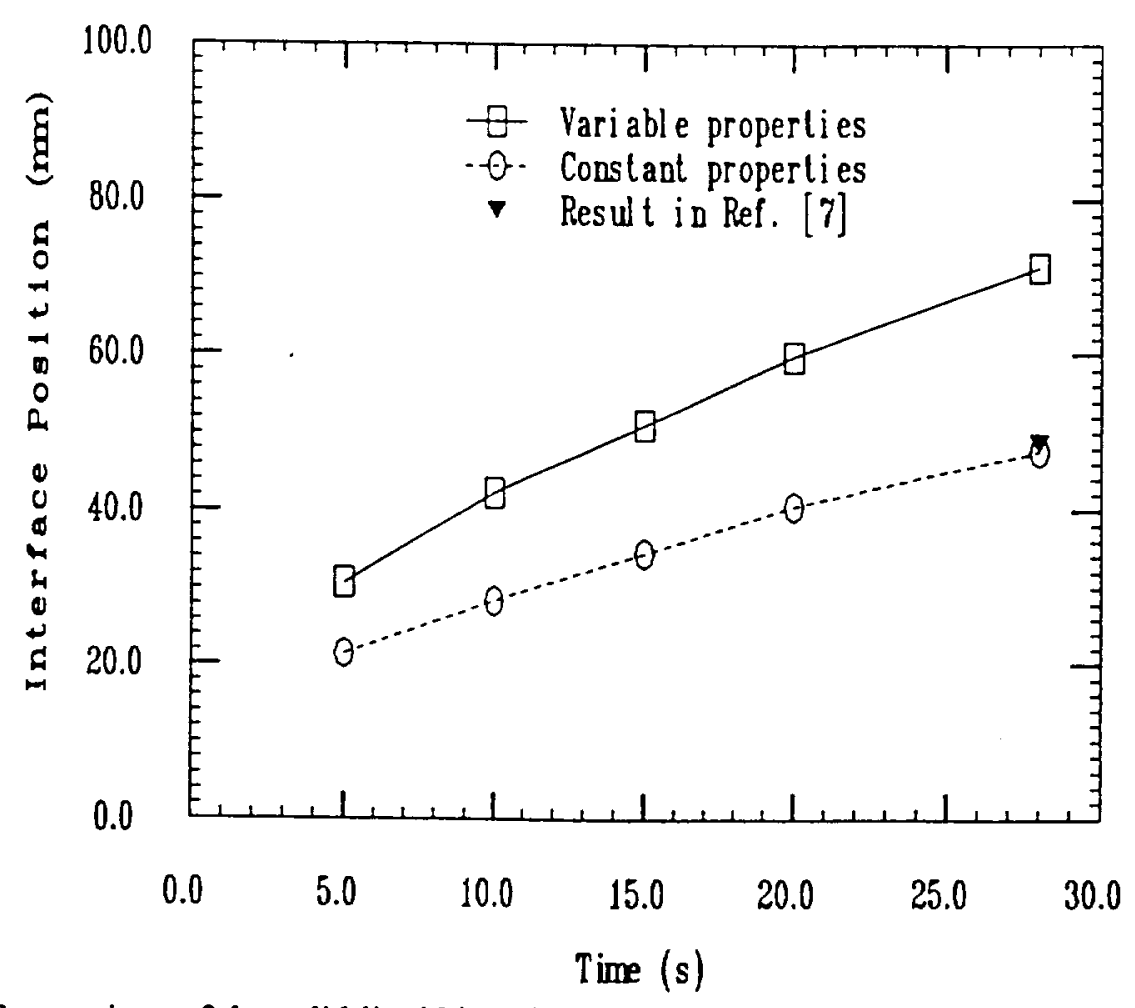

Figure 6: Comparison of the solid-liquid interface positions for liquid aluminum solidification process, both conduction and natural convection are considered

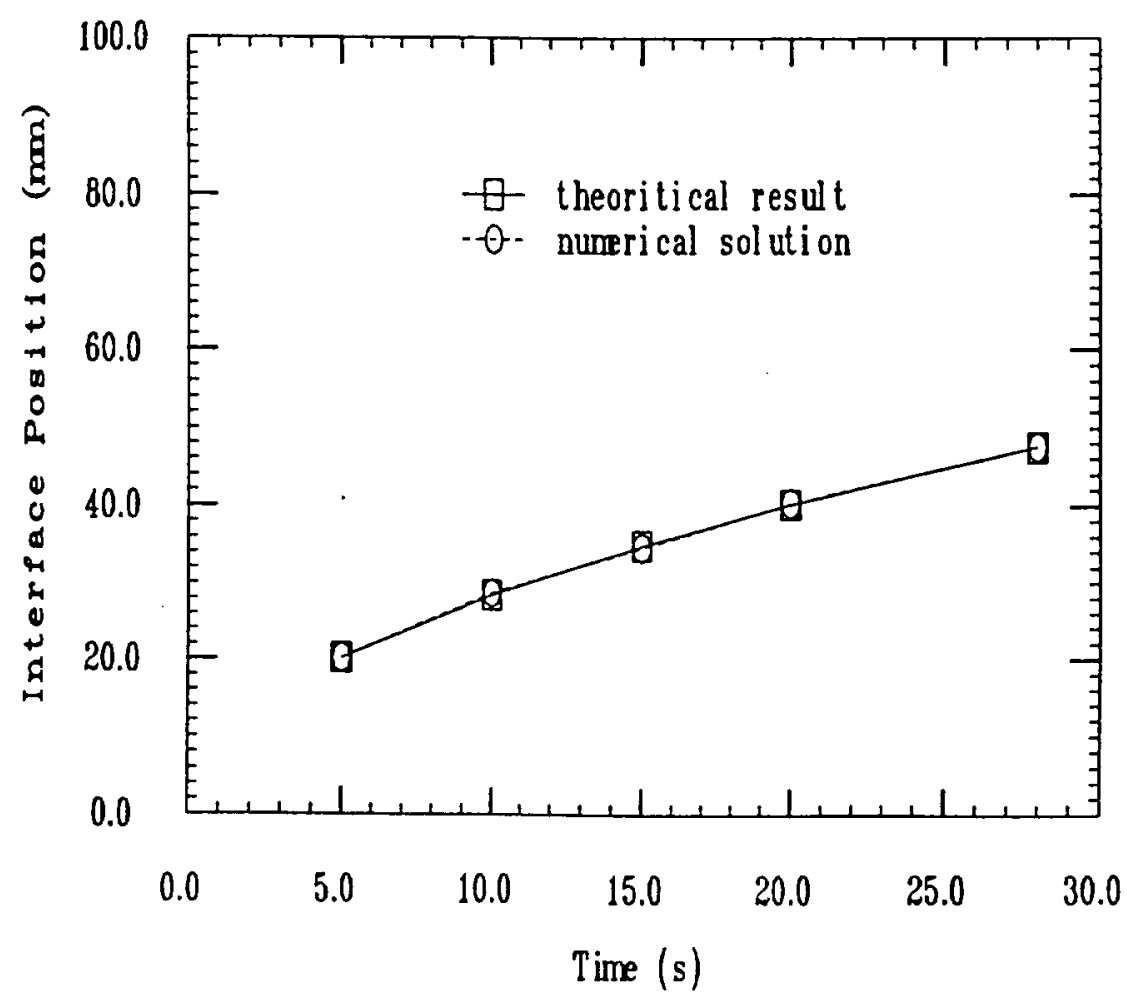

Figure 7: Comparison of the solid-liquid interface positions for liquid aluminum solidification process, constant properties and conduction only 